\title{
Cardiac Arrest and Death in Neurosurgery: An Analysis of Perioperative Anesthetic Adverse Events in Thailand
}

\author{
Phuping Akavipat ${ }^{1}$ Pimwan Sookplung ${ }^{1}$ Thanatporn Boonsombat ${ }^{1}$ Manee Raksakietisak ${ }^{2}$ \\ Cataleya Tongrong ${ }^{3}$ Surunchana Lerdsirisopon ${ }^{4} \quad$ Varinee Lekprasert $^{5} \quad$ Yodying Punjasawasdwong $^{6}$ \\ Vichai Ittichaikulton ${ }^{5}$
}

${ }^{1}$ Department of Anesthesiology, Prasat Neurological Institute, Bangkok, Thailand

2Department of Anesthesiology, Siriraj Hospital, Mahidol University, Bangkok, Thailand

${ }^{3}$ Department of Anesthesiology, Srinakarind Hospital, Khon Kaen University, Khon Kaen, Thailand

${ }^{4}$ Department of Anesthesiology, King Chulalongkorn Memorial

Hospital, Chulalongkorn University, Bangkok, Thailand

${ }^{5}$ Department of Anesthesiology, Ramathibodi Hospital, Mahidol

University, Bangkok, Thailand

${ }^{6}$ Department of Anesthesiology, Maharaj Nakorn Chiangmai

Hospital, Chiangmai University, Chiangmai, Thailand

\author{
Address for correspondence Phuping Akavipat, MD, FRCAT, PhD, \\ Department of Anesthesiology, Prasat Neurological Institute, \\ 312 Rajvithee Road, Bangkok 10400, Thailand \\ (e-mail: ppakvp@hotmail.com).
}

J Neuroanaesthesiol Crit Care 2018;5:87-93.

\begin{abstract}
Keywords

- complication

- risk

- cardiopulmonary resuscitation

- neuroanesthesia

- national audit

Background Complexities of pathological causes in cardiac arrest and death in neurosurgery require individualized management. To decrease the incidents, this study was performed to describe characteristics and factors reducing adverse outcomes together with potential corrective strategies of perioperative cardiac arrest and death in neurosurgical patients.

Methods An observational study was performed in 22 hospitals. Peer-reviewed consensus was formed using database from Perioperative Anesthetic Adverse Events in Thailand (PAAd Thai) study. The data contain demography, anesthetic, surgical details, opinions on contributing factors, and factors that minimized incident as well as suggested corrective strategies.

Results From 2,000 incidents, 64 (3\%) cardiac arrest events were reported with a 50\% chance of return of spontaneous circulation. The most common cardiac rhythm documented was asystole. Essentially, electrocardiography was the most frequent early detector. Surgical-related factors (81.3\%), mostly intraoperative bleeding, played a major role in potential causes of arrest. In addition, anesthesia, patient conditions, and system-related factors were found in $65.6 \%, 57.8 \%$, and $8.3 \%$ of the incidents, respectively. Moreover, the severity of the patients (73.3\%) was considered to be the most common anesthesia-related risk factor. The prevention of an incident included rule enforcement for patient safety, mandatory knowledge, and also anesthesia personnel's skills development.

Conclusion The optimum fluid assessment and resuscitation, cerebral protection protocols, clinical awareness, and quality assurance together with human resource management are all essential to eliminate the catastrophic cardiac arrest and death in neurosurgery.
\end{abstract}

Copyright $\odot 2018$ Indian Society of Neuroanaesthesiology and Critical Care

\section{License terms}

() (1) $\ominus \circledast$ 


\section{Introduction}

Although a cardiac arrest or a death in neurosurgery does not occur frequently, an impact of an occurrence is severe enough to alter an anesthetic practice and to affect the judgment of a practitioner. The altered practice and judgment are proceeded to avoid a cardiac arrest that leads to severe brain dysfunction from global-ischemic brain injury. ${ }^{1}$ According to a survey in 2005, Thai Anesthesia Incidents Study (THAI Study), which was the first national statistical data of mortality and morbidity related to anesthesia ( $n=163,403)$, reported an overall perioperative cardiopulmonary arrest within 24 hours of 30.8:10,000 and death of 28.3:10,000. ${ }^{2}$ In addition, an incident report in 2008 showed $14 \%$ cardiopulmonary arrest from 2,537 incidents. ${ }^{3}$ Approximately a decade later, after risk management strategies initiated by the Royal College of Anesthesiologists of Thailand were deployed, the incidence decreased significantly to $15.6: 10,000$ for the perioperative cardiac arrest and 13.3:10,000 for perioperative death. ${ }^{4}$ Even though the incidence has reduced significantly, continuously available literature monitoring and updating warrant prevention of perioperative cardiac arrest strategy or recommendation is still essential.

Every cardiac arrest in neurosurgical patients is complex and unique. Neurosurgical patients have certain factors that influence cardiopulmonary resuscitation (CPR) especially on mechanisms of cardiac arrest, for example intracranial pathology, surgical procedure, and position of the patient., The individual management based on anesthetic knowledge to enhance safety and improve the outcomes should be integrated properly, for example patient monitoring, dealing with unexpected events, personal nontechnical skill and human resource allocation, etc. Therefore, with a collaboration of the Royal College of Anesthesiologists of Thailand, this study aimed to investigate incidences, characteristics, related factors that minimize adverse outcomes, and suggestive strategies for cardiac arrest and death in neurosurgery in 2015.

\section{Materials and Methods}

This study was approved by the Prasat Neurological Institutional Ethics Committee on February 20, 2017 (Number 014/2560; Chairman: Suchart Hanchaipiboonkul) and registered to the Thai Clinical Trials Registry (TCTR) with the identification number of TCTR20170503001.

With the collaboration of 22 hospitals resided in Thailand, a prospective and multicenter observational study of potential causes and corrective strategies of perioperative cardiac arrest and death in neurosurgical patients was conducted. Standard incident record forms were anonymously filled out by performing anesthesiologists or related anesthesia personnel as a part of Perioperative Anesthetic Adverse Events in Thailand (PAAd Thai) study from January 1 to December 31, 2015. Perioperative cardiac arrest and death data were collected and reviewed. According to the definition, perioperative cardiac arrest and death were defined as cardiopulmonary arrest or death occurred in the hospital, which practically needs $\mathrm{CPR}$, not including a temporary beating stop from surgical stimuli during intraoperative period and the first 24 hours postoperatively. The records contained demographic data, anesthetic, surgical details, opinions on factors influencing an unfavorable event, and factors minimizing incident as well as suggested corrective strategies.

Thereafter, the standard record forms were thoroughly reviewed by three experienced anesthesiologists and clinical epidemiologist to create a consensus on root-cause analysis. The analysis was performed to identify the most likely etiology of the events categorized into characteristics of patients, methods of surgery, anesthetic and system factors, proper initial management, and also developments of suitable preventive strategies.

In the next session, a descriptive statistical analysis is presented. The continuous data are represented in means \pm standard deviation (SD) whereas categorical data are shown in numbers and percentage.

\section{Results}

From 2,000 records, there were 60 (3.0\%) patients with 64 events of cardiac arrest during perioperative neurosurgery under anesthesia services. The average age was $49.65 \pm 17.71$ years with the operation time of $135.86 \pm 115.46$ minutes. The majority of patients $(41 ; 68.3 \%)$ had cardiac arrest in the medical service-directed hospital whereas the rest (19; $31.7 \%$ ) were in the academic-directed hospitals. Most of the patients arrested in supine position (59; 98.3\%). Balanced anesthetic technique with inhalation agent either sevoflurane (33; $84.6 \%)$, isoflurane $(5 ; 12.8 \%)$, or desflurane $(1 ; 2.6 \%)$ was used in $39(65.0 \%)$ patients. Moreover, total intravenous anesthesia was given in 21 (35.0\%) patients with propofol (8; $38.1 \%)$, narcotics $(6 ; 28.6 \%)$, thiopental $(4 ; 19.1 \%)$, midazolam (2; 9.5\%), and sole muscle relaxant (1; $4.8 \%)$. Nitrous oxide was added in seven (17.9\%) cases of general anesthesia with inhalation agents. The demographic of the cardiac arrest patients is demonstrated in - Table 1.

There were 24 (40.0\%) incidents that could have been prevented. The prevention can include (1) rule enforcement in three (12.5\%) cases, that is, prohibiting practices of supervised anesthetic personnel in patients with ASA (American Society of Anesthesiologists) physical status > III and limiting the number of supervised cases to one anesthesiologist at a time; (2) supplementary knowledge in 18 (75.0\%) cases, that is, knowledge of brain protection strategy, pathology of severe brain injury, massive blood transfusion management, and advanced neuromonitoring; and (3) improved administrative skills in 13 (54.2\%) cases, that is, awareness of potential massive bleeding and anesthetists' nontechnical skills (ANTS). Among these preventions, only seven (11.7\%) events were related to the World Health Organization (WHO) surgical safety checklist.

According to the CPR procedures, all the clinical treatments were acceptable. Forty (62.5\%) medical staffs who detected these critical events were anesthetic personnel, 18 (28.1\%) were registered nurses, and 6 (9.4\%) were surgeons. The defibrillation was done in six (9.4\%) patients who had fulfilled indication. From 64 arrests, the "do not attempt to resuscitate" (DNAR) 
Table 1 Demographics and anesthetic details of the cardiac arrest patients undergoing neurosurgery

\begin{tabular}{|c|c|c|}
\hline & Number & Percent \\
\hline \multicolumn{3}{|l|}{ Sex } \\
\hline Male & 24 & 40.0 \\
\hline Female & 36 & 60.0 \\
\hline \multicolumn{3}{|l|}{ ASA physical status } \\
\hline$I-I \mid$ & 7 & 11.7 \\
\hline III-V & 53 & 88.3 \\
\hline \multicolumn{3}{|l|}{ Diagnosis } \\
\hline Cerebral tumor & 7 & 11.7 \\
\hline Cerebral aneurysm & 2 & 3.3 \\
\hline Traumatic brain injury & 48 & 80.0 \\
\hline Hydrocephalus & 2 & 3.3 \\
\hline Spinal fracture & 1 & 1.7 \\
\hline \multicolumn{3}{|l|}{ Operation } \\
\hline Craniotomy with tumor removal & 7 & 11.6 \\
\hline Craniotomy with aneurysm clipping & 2 & 3.3 \\
\hline Craniotomy with remove blood clot & 19 & 31.7 \\
\hline Craniectomy with remove blood clot & 19 & 31.7 \\
\hline Ventriculostomy & 9 & 15.0 \\
\hline Shunt & 3 & 5.0 \\
\hline Spinal surgery & 1 & 1.7 \\
\hline Emergency situation & 48 & 80.0 \\
\hline Nonofficial hours & 42 & 70.0 \\
\hline \multicolumn{3}{|l|}{ Anesthetic techniques } \\
\hline Balanced technique with inhalation anesthesia & 39 & 65.0 \\
\hline Balanced technique with total intravenous anesthesia & 21 & 35.0 \\
\hline \multicolumn{3}{|l|}{ Monitoring } \\
\hline Arterial blood pressure & 20 & 33.3 \\
\hline Central venous pressure & 12 & 20.0 \\
\hline End-tidal gas tension & 29 & 48.3 \\
\hline End-tidal carbon dioxide tension & 57 & 95.0 \\
\hline \multicolumn{3}{|l|}{ Anesthesia performers } \\
\hline Certified anesthesiologists & 35 & 58.3 \\
\hline Nurse anesthetists under supervision & 17 & 28.3 \\
\hline Anesthetic residents under supervision & 8 & 13.3 \\
\hline
\end{tabular}

was preordered in the intensive care unit (ICU) for 11 (36.7\%) arrests, in the surgical ward for 18 (60.0\%) arrests, and in the operating theater for 1 (3.3\%) arrest. Nevertheless, after excluding the events of cardiopulmonary arrest with DNAR order, the return of spontaneous circulation (ROSC) appeared in 17 (50.0\%) patients, that is, in the operating theater for 11 (64.7\%) patients, in the ICU for 5 (29.4\%) patients, and in surgical ward for 1 (5.9\%) patient. Adrenaline was used in all occurrences. Dopamine, noradrenaline, atropine, sodium bicarbonate, and calcium were given 11 (32.4\%), 3 (8.8\%), 7 (20.6\%), 13 (38.2\%), and 6 (17.6\%) arrests, respectively. The mean \pm SD duration of the procedure was $18.5 \pm 32.4$ minutes, and particularly in the ROSC case it was $5 \pm 6.8$ minutes. The characteristics of CPR procedures in total are shown in - Table 2.

The Glasgow outcome scale at a hospital discharge shows score of 1 (nonsurvivor) in 55 (91.7\%) patients, 2 (severe disability) in 1 (1.7\%) patient, and 3-5 (moderate disability to no disability) in 4 (6.7\%) patients. Three deaths (5.8\%) were declared in the operating theater, $19(36.5 \%)$ in the ICU, and 30 (57.7\%) in the surgical ward.

The majority of cardiac arrest events $(52 ; 81.3 \%)$ related to a surgery, the other possible causes were due to anesthesia (42; 65.6\%), patient-related (37; 57.8\%), and system-related $(30 ; 46.9 \%)$, as demonstrated in - Table 3. 
Table 2 The characteristics of cardiopulmonary resuscitation procedures

\begin{tabular}{|c|c|c|}
\hline & Number & Percent \\
\hline \multicolumn{3}{|l|}{ Period } \\
\hline Intraoperative periods & 15 & 23.4 \\
\hline Postoperative periods & 49 & 76.6 \\
\hline \multicolumn{3}{|l|}{ Primary method of recognition } \\
\hline Clinical recognition prior to monitor & 9 & 14.0 \\
\hline Electrocardiography & 30 & 46.9 \\
\hline Noninvasive blood pressure monitor & 18 & 28.1 \\
\hline Arterial blood pressure monitor & 4 & 6.3 \\
\hline Plethysmography & 3 & 4.7 \\
\hline \multicolumn{3}{|l|}{ Initial cardiac rhythms } \\
\hline Asystole & 23 & 35.9 \\
\hline Pulseless electrical activity & 7 & 10.9 \\
\hline Bradycardia & 3 & 4.7 \\
\hline Ventricular fibrillation & 3 & 4.7 \\
\hline Others & 1 & 1.6 \\
\hline Unknown & 27 & 42.2 \\
\hline Witness arrest & 64 & 100.0 \\
\hline "Do not attempt to resuscitate" order & 30 & 46.9 \\
\hline
\end{tabular}

Table 3 The possible causes of cardiac arrest in neurosurgical patients

\begin{tabular}{|c|c|c|}
\hline & Number & Percent \\
\hline \multicolumn{3}{|l|}{ Surgical related } \\
\hline Intraoperative bleeding & 21 & 32.8 \\
\hline Cerebral edema & 17 & 26.6 \\
\hline Postoperative bleeding & 12 & 18.8 \\
\hline Skull fracture & 2 & 3.1 \\
\hline \multicolumn{3}{|l|}{ Anesthesia related } \\
\hline Cardiovascular instability/hypotension & 34 & 53.1 \\
\hline Airway problems ${ }^{\mathrm{a}}$ & 4 & 6.3 \\
\hline Pulmonary problems ${ }^{\mathrm{b}}$ & 4 & 6.3 \\
\hline \multicolumn{3}{|l|}{ Patient related } \\
\hline Severe brain damage & 21 & 32.8 \\
\hline Metabolic derangement & 9 & 14.1 \\
\hline Lethal arrhythmia & 4 & 6.3 \\
\hline Miscellaneous & 3 & 4.7 \\
\hline \multicolumn{3}{|l|}{ Systemic related } \\
\hline Incoordination & 4 & 6.3 \\
\hline Intensive care unit utilization pitfall & 26 & 40.6 \\
\hline
\end{tabular}

${ }^{a}$ Airway problems: airway injury, obstruction.

bPulmonary problems: aspirated pneumonitis, pneumonia, pulmonary embolism, traumatic lung injury.

Intraoperative cardiac arrests were intraoperative bleeding and led to severe hypotension (14: 93.3\%), tight brain (14: $93.3 \%)$, severe brain damage (12: $80 \%$ ), severe metabolic acidosis (4: $26.7 \%$ ), lethal arrhythmia (1: 6.7\%), and other (2: $13.3 \%$ ). The emergency cross-matching for blood products was documented during 5 (8.3\%) surgical procedures. Additionally, one (1.7\%) particular cause of death was referred to pulmonary embolism.

- Table 4 presents contributing factors, factors minimizing the incidences, and suggestive corrective strategies. 
Table 4 Contributing factors, factors minimizing the events, and suggestive corrective strategies for the cardiac arrest in neurosurgical patients

\begin{tabular}{|c|c|c|}
\hline & Number & Percent \\
\hline \multicolumn{3}{|l|}{ Contributing factors } \\
\hline Noncompliance to WHO surgical safety checklist & 3 & 5.0 \\
\hline Inappropriate decision making & 25 & 41.7 \\
\hline Lack of knowledge & 25 & 41.7 \\
\hline Inexperience & 24 & 40.0 \\
\hline Emergency situation & 48 & 80.0 \\
\hline Shortage of manpower & 5 & 8.3 \\
\hline Improper patient evaluation and preparation & 33 & 55.0 \\
\hline Lack of monitoring equipment & 16 & 26.7 \\
\hline Mismatched blood transfusion & 4 & 6.7 \\
\hline Communication mishaps & 17 & 28.3 \\
\hline Improper postoperative care unit & 29 & 48.3 \\
\hline Patient severity & 44 & 73.3 \\
\hline \multicolumn{3}{|l|}{ Factor minimizing the event } \\
\hline Comply to checklist and guideline & 21 & 35.0 \\
\hline Previous experienced & 41 & 68.3 \\
\hline Capable assistant & 37 & 61.7 \\
\hline Clinical awareness & 46 & 76.7 \\
\hline Effective consultation system & 33 & 55.0 \\
\hline Competent training & 7 & 11.7 \\
\hline Effective patient monitoring systems & 17 & 28.3 \\
\hline \multicolumn{3}{|l|}{ Suggestive corrective strategies } \\
\hline Develop treatment guideline and recommendations & 36 & 60.0 \\
\hline Appropriated human resource management enterprise & 46 & 76.7 \\
\hline Efficient supervision & 37 & 61.7 \\
\hline Quality control of medical equipment & 15 & 25.0 \\
\hline Quality assurance activity & 56 & 93.3 \\
\hline Intensive care utilization analysis & 12 & 20.0 \\
\hline
\end{tabular}

Abbreviation: WHO, World Health Organization.

The recommendations to decrease intraoperative mortality included mainly promoting quality assurance program (15: 100\%); developing clinical practice guidelines (14: 93.3\%) such as massive blood transfusion protocol, cerebral protection regimen, and tight brain management paradigm; and intensifying effective medical personnel control (9: 60.0\%). Appropriate human resource management is categorized into enhancement of performance and competency (43; 71.7\%), educational achievements in clinical anesthesia (20; 33.3\%), and personnel allocations (19; 31.7\%) whereas enhancement of performance and competency is divided into vigilance to protocols $(10 ; 23.3 \%)$ and effective communication (33; 76.7\%).

\section{Discussion}

This article is a part of the PAAd Thai study that is the third national closed-claim study of the eventful complications perioperatively. ${ }^{4,7}$ The report is based on a voluntary and anonymous basis as a multicenter trial. The incidences were collected at a shared central database but analyzed separately according to individual working group interest. The definitions of each complication were specified in the prerequisite document, and the consensus method was used for the conclusion of the contributing factors, the factors minimizing the event, and the suggestive corrective strategies.

To the best of our knowledge, this is the most recent primary report concerning perioperative cardiac arrest in neurosurgery. Therefore, we do not have a benchmark to compare the occurrences. Even though patient's age, ASA physical status classification, duration of operation, hospital type, emergency situation, diagnosis, and activities during nonofficial hour seem to be common causes of cardiac arrests in general, ASA physical status III-V, emergency condition, and absence or no monitoring of capnometer can also be considered risk factors for perioperative death in intracranial 
surgery. However, the anesthetic technique and anesthetic agents are not mentioned in Brovman et al, ${ }^{8}$ Zajac, ${ }^{9}$ Watters et al, ${ }^{10}$ Zhou, ${ }^{11}$ and Akavipat. ${ }^{12}$

Among in-hospital cardiac arrest patients, pulseless electrical activity was the most frequent cause of death from the entire mechanism of fatal arrhythmias. ${ }^{13}$ Though this study result differed, cardiac standstill was observed as the firstrank cause particularly in neurosurgical patients. Pathology of autonomic nervous system in higher brain center accompanying with surgical stimulations is discussed. ${ }^{14} \mathrm{~A}$ critical issue is how to detect this lethal phenomenon as early as possible. Either clinical recognition or information from monitoring equipment would be the best for an incident warning system..$^{15}$ The preceding electrocardiographic changes in either the rhythms or the morphology might alert the medical personnel before it converts to a cardiac arrest. ${ }^{13}$ However, how well are the observers aware? This question has no answer yet. The supervision system, presence of a board-certified anesthesiologist, continuing medical education, and training in the aspect of ANTS are proposed in Brovman et al, ${ }^{8}$ Chang, ${ }^{16}$ and Lee et al. ${ }^{17}$

In this report, the ROSC and the survived patients are higher than the other, reflected fast recognition and abrupt chest compression without any doubts in initial rhythm. ${ }^{18}$ Besides the initial cardiac rhythm, the amount of blood loss, the CPR duration and the episodes of hypotension are also demonstrated as the factors related to the ROSC. ${ }^{19,20}$

The causes of death are principally severe brain damage and cardiovascular and hemodynamics mishaps. Nonetheless, a cerebral protection scheme is not acknowledged documentarily to decrease mortality as well as an intraoperative volume assessment and an effective fluid resuscitation. ${ }^{21}$ Most of the threats that have been discussed are delayed responsiveness. The intraoperative fluid resuscitation including the intravascular volume assessment should be performed continually to minimize this complication as well as the initiation of contingency diagram to deal with intraoperative brain swelling. The institutional massive blood transfusion policy should be declared and followed restrictively emphasizing the criteria to activate this emergency protocol at the appropriated point of time. ${ }^{22,23,24,25}$ Another interesting issue is referred to the ICU utilization. Approximately, onethird of the critically ill patients who have no DNAR order are shifted to the surgical ward instead of an ICU because of an error in judgment, an administrative mistake, or miscommunication. The way of monitoring, critical approaching, and therapeutic facilities might differ. Hence, deaths can be potentially prevented at this level. ${ }^{26}$

Surprisingly, WHO surgical safety checklist seems to be less useful for the factors minimizing cardiac arrest in neurosurgery although it is found favorable in general surgeries. ${ }^{27}$ Characteristics of neurosurgical patients and operative procedures are unique. ${ }^{28} \mathrm{Clinical}$ responsiveness following a treatment is extremely dynamic in these fragile patients. The possibility of decreasing cardiac arrest events does not depend predominantly on systematic problem recognition, risk management, or communication skills that can be revealed by the WHO surgical safety checklist. However, the authors would still recommend accomplishing the WHO surgical safety checklist routinely to prevent unpredictability and other complications.

Structure of anesthesiology department, especially logistics and personnel, was remarked as a cofactor in perioperative mortality. ${ }^{17}$ Clinical awareness, reasonable judgment, working experience, treatment guideline/recommendation, and knowledge integration are identified as suggestive corrective strategies based on the current study. The scenarios to manage this dilemma were listed elsewhere as debriefing after simulation-based nontechnical skill training, operational awareness, and medical professionalism..$^{29,30}$

The limitation of this study is the methodology itself. Because the retrospective data are collected on voluntary and anonymous basis, some reports are probably questionable. The quality control of data management and the rigorous action of individual site managers would be beneficial. In conclusion, perioperative cardiopulmonary arrests during anesthesia in neurosurgery were found $3 \%$ from the overall 2,000 patients who had noteworthy adverse events. Pitfalls in intraoperative fluid assessment and resuscitation as well as cerebral protection strategies were remarkable in potentially preventable incidents. Encouragement of clinical awareness, quality assurance, and appropriate human resource management were the effective means for the risk reduction.

\section{Funding Support}

Royal College of Anesthesiologists of Thailand, Prasat Neurological Institute, Faculty of Medicine of Chiang Mai University, Chulalongkorn University, Khon Kaen University, Mahidol University (Siriraj Hospital and Ramathibodi Hospital), Prince of Songkla University, Health System Research Institute, and National Research Council of Thailand.

\section{Conflict of Interest}

None.

\section{Acknowledgments}

This multicenter study was accomplished by personal sacrifice, inspiration together with the cooperation among Thai anesthesiologists, the Anesthesiology Department directors of the participating sites, and the Thai Society for Neuroanesthesia. The Royal College of Anesthesiologists of Thailand (RCAT) and the PAAd Thai study group wish to express deep gratitude to project advisors Professor Thara Tritrakarn; Professor Somsri Paosawasdi; Associate Professor Khun Wanna Somboonwiboon; and Associate Professor Oranuch Kyokong for their exceptionally encouragement, suggestion, and advices.

Furthermore, the authors would like to extend the appreciation to Professor Somrat Charuluxananan, Associate Professor Yodying Punjasawadwong for their devotion and also for the language revision. 


\section{References}

1 Ellis SJ, Newland MC, Simonson JA, et al. Anesthesia-related cardiac arrest. Anesthesiology 2014;120(4):829-838

2 Charuluxananan S, Punjasawadwong Y, Suraseranivongse S, et al. The Thai Anesthesia Incidents Study (THAI Study) of anesthetic outcomes: II. Anesthetic profiles and adverse events. J Med Assoc Thai 2005;88Suppl 7):S14-S29

3 Charuluxananan S, Suraseranivongse S, Jantorn P, et al. Multicentered study of model of anesthesia related adverse events in Thailand by incident report (The Thai Anesthesia Incidents Monitoring Study): results. J Med Assoc Thai 2008;91(7):1011-1019

4 Charuluxananan S, Sriraj W, Punjasawadwong Y, et al. Perioperative and anesthetic adverse events in Thailand (PAAD THAI) incident reporting study: anesthetic profiles and outcomes. Asian Biomed 2017;11(1):21-32

5 McClain CD, Soriano SG. Anesthesia for intracranial surgery in infants and children. Curr Opin Anaesthesiol 2014;27(5):465-469

6 Chowdhury T, Prabhakar H, Bithal PK, Schaller B, Dash HH. Immediate postoperative complications in transsphenoidal pituitary surgery: A prospective study. Saudi J Anaesth 2014;8(3):335-341

7 Punjasawadwong Y, Sriraj W, Charuluxananan S, et al. Perioperative and anesthetic adverse events in Thailand (PAAD THAI) incident reporting study: hospital characteristics and methods. Asian Biomed 2017;11(1):33-39

8 Brovman EY, Gabriel RA, Lekowski RW, Dutton RP, Urman RD. Rate of major anesthetic-related outcomes in the intraoperative and immediate postoperative period after cardiac surgery. J Cardiothorac Vasc Anesth 2016;30(2):338-344

9 Zajac K, Zajac M. Perioperative mortality. Przegl Lek 2005; 62(3):173-180

10 Watters DA, Babidge WJ, Kiermeier A, McCulloch GA, Maddern GJ. Perioperative mortality rates in australian public hospitals: the influence of age, gender and urgency. World J Surg 2016;40(11):2591-2597

11 Zhou Y, Li W, Herath C, et al. Off-hour admission and mortality risk for 28 specific diseases: a systematic review and metaanalysis of 251 cohorts. J Am Heart Assoc 2016;5(3):e003102

12 Akavipat P, Ittichaikulthol W, Tuchinda L, Sothikarnmanee T, Klanarong S, Pranootnarabhal T. The Thai Anesthesia Incidents (THAI Study) of anesthetic risk factors related to perioperative death and perioperative cardiovascular complications in intracranial surgery. J Med Assoc Thai 2007;90(8):1565-1572

13 Do DH, Hayase J, Tiecher RD, Bai Y, Hu X, Boyle NG. ECG changes on continuous telemetry preceding in-hospital cardiac arrests. J Electrocardiol 2015;48(6):1062-1068

14 Biering-Sørensen F, Biering-Sørensen T, Liu N, Malmqvist L, Wecht JM, Krassioukov A. Alterations in cardiac autonomic control in spinal cord injury. Auton Neurosci 2018;209:4-18

15 Bein B, Seewald S, Gräsner JT. How to avoid catastrophic events on the ward. Best Pract Res Clin Anaesthesiol 2016;30(2):237-245
16 Chang $\mathrm{CH}$. Medical simulation is needed in anesthesia training to achieve patient's safety. Korean J Anesthesiol 2013;64(3):204-211

17 Lee JH, Kim EK, Song IK, et al. Critical incidents, including cardiac arrest, associated with pediatric anesthesia at a tertiary teaching children's hospital. Paediatr Anaesth 2016;26(4):409-417

18 Amnuaypattanapon K, Udomsubpayakul U. Evaluation of related factors and the outcome in cardiac arrest resuscitation at Thammasat Emergency Department. J Med Assoc Thai 2010;93Suppl 7):S26-S34

19 Fei Y, Wang L, Zhong T. Factor analysis for the prognosis of perioperative cardiopulmonary resuscitation [in Chinese] Zhonghua Yi Xue Za Zhi 2014;94(1):18-21

20 Yi HJ, Kim YS, Ko Y, Oh SJ, Kim KM, Oh SH. Factors associated with survival and neurological outcome after cardiopulmonary resuscitation of neurosurgical intensive care unit patients. Neurosurgery 2006;59(4):838-845, discussion 845-846

21 Germans MR, Boogaarts HD, Macdonald RL. Neuroprotection in critical care neurology. Semin Neurol 2016;36(6):642-648

22 Cleland S, Corredor C, Ye JJ, Srinivas C, McCluskey SA. Massive haemorrhage in liver transplantation: consequences, prediction and management. World J Transplant 2016;6(2):291-305

23 Tran A, Matar M, Steyerberg EW, Lampron J, Taljaard M, Vaillancourt C. Early identification of patients requiring massive transfusion, embolization, or hemostatic surgery for traumatic hemorrhage: a systematic review protocol. Syst Rev 2017;6(1):80

24 Wijdicks EF, Sheth KN, Carter BS, et al; American Heart Association Stroke Council. Recommendations for the management of cerebral and cerebellar infarction with swelling: a statement for healthcare professionals from the American Heart Association/American Stroke Association. Stroke 2014;45(4):1222-1238

25 Treml AB, Gorlin JB, Dutton RP, Scavone BM. Massive transfusion protocols: a survey of Academic Medical Centers in the United States. Anesth Analg 2017;124(1):277-281

26 Teixeira PG, Inaba K, Hadjizacharia P, et al. Preventable or potentially preventable mortality at a mature trauma center. J Trauma 2007;63(6):1338-1346, discussion 1346-1347

27 Haugen AS, Søfteland E, Almeland SK, et al. Effect of the World Health Organization checklist on patient outcomes: a stepped wedge cluster randomized controlled trial. Ann Surg 2015;261(5):821-828

28 Ibrahim GM, Morgan BR, Macdonald RL. Patient phenotypes associated with outcomes after aneurysmal subarachnoid hemorrhage: a principal component analysis. Stroke 2014;45(3):670-676

29 Garden AL, Le Fevre DM, Waddington HL, Weller JM. Debriefing after simulation-based non-technical skill training in healthcare: a systematic review of effective practice. Anaesth Intensive Care 2015;43(3):300-308

30 Matveevskii A, Moore DL, Samuels PJ. Competency and professionalism in medicine. Clin Teach 2012;9(2):75-79 